\title{
PRODUTIVIDADE DE CULTIVARES DE CAFEEIROS (Coffea arabica L.) SUBMETIDAS A DIFERENTES ÉPOCAS DE PARCELAMENTO DA ADUBAÇÃO ${ }^{1}$
}

\author{
GABRIEL FERREIRA BARTHOLO ${ }^{2}$ \\ PAULO TÁCITO GONTIJO GUIMARÃES ${ }^{2}$ \\ ANTÔNIO NAZARENO GUIMARÃES MENDES ${ }^{3}$
}

\begin{abstract}
RESUMO - Os experimentos foram instalados na Fazenda Experimental da Epamig em São Sebastião do Paraíso, com o objetivo de estudar o comportamento das cultivares Mundo Novo-IAC 379/19; Icatu PrecoceIAC 3282; Icatu Amarelo-IAC 2944 e Rubi-MG 1192, em relação a épocas de parcelamento de adubação. As respostas das cultivares foram medidas sobre a produção em função das épocas de parcelamento da adubação, demonstrando que a característica estudada foi influenciada pelas combinações das épocas em que foram
\end{abstract}

submetidas. A cultivar Mundo Novo-IAC 379/19 tolerou intervalos maiores entre as adubações no período de outubro a março, e a cultivar Icatu Amarelo-IAC 2944 respondeu de modo significativo a quatro parcelamentos consecutivos com intervalo de 30-40 dias entre as aplicações. Para a cultivar Icatu Precoce-IAC 3282, existem opções de estabelecimento de épocas adequadas, dependendo do início das chuvas. A cultivar Rubi-MG 1192 não respondeu às épocas de parcelamento da adubação.

TERMOS PARA INDEXAÇÃO: Parcelamento, adubação, cafeeiro, Coffea arabica.

\section{YIELD OF COFFEE CULTIVARS (Coffea arabica L.) UNDER DIFFERENT SPLIT-FERTILIZATION TIMES}

\begin{abstract}
The work was conducted at EPAMIG's Experimental Station in São Sebastião do Paraíso, MG, to study the performance of Mundo Novo-IAC 379/19; Icatu Precoce-IAC 3282; Icatu Amarelo-IAC 2944 and RubiMG 1192 coffee cultivars, in relation to time and parceling out of fertilization. Cultivar responses were evaluated by yield measurements, with the results showing that this parameter was affected by time in which fertilization was
\end{abstract}

made. Mundo Novo-IAC 379/19 cultivar tolerated broader intervals between the fertilizations from October to March. Icatu Amarelo-IAC 2944 response was significant to four consecutive parceling, with intervals from 30 to 40 days between applications. Icatu Precoce-IAC 3282 cultivar presented other more appropriate time options depending on the start of rainy season. Rubi-MG 1192 cultivar was not responsive to split-fertilization.

INDEX TERMS: Split-fertilizing, fertilizing, coffee, Coffea arabica.

\section{INTRODUÇÃO}

A cafeicultura brasileira em quase sua totalidade está situada ao sul do paralelo $20^{\circ} \mathrm{S}$. Essa condição tropical de altitude resulta num ciclo térmico bem acentuado. Como resultado, os cafeeiros exibem um ritmo fenológico bem determinado, com florescimento na primavera, frutificação no verão, maturação no outono e repouso no inverno. Essas características climáticas e fenológicas da cafeicultura nacional devem ser consideradas para melhor utilização dos nutrientes pelas plantas por meio das adubações (Miguel, 1978).

Entretanto, o fracionamento das doses de nitrogênio $(\mathrm{N})$ em razão das necessidades momentâneas da planta não é utilizado. O que é feito comumente é o parcelamento do $\mathrm{N}$ em partes iguais de setembro a março e, muitas vezes, aplicado depois que o fruto atingiu 90 dias (Miguel, 1978).

1. Parte da tese apresentada à UNIVERSIDADE FEDERAL DE LAVRAS, Caixa Postal 37 - 37200-000 - Lavras, $M G$, pelo primeiro autor, para obtenção do titulo de Doutor em Agronomia, área de concentração, Fitotecnia.

2. Engenheiro Agrônomo D.S., Pesquisador da EPAMIG, CTSM, Caixa Postal 176 - 37.200-000, Lavras, MG.

3. Engenheiro Agrônomo, D.S., Professor Titular do Departamento de Agricultura/UFLA. 
Como o parcelamento do $\mathrm{N}$ é condição essencial para um melhor aproveitamento do fertilizante e uma boa produção, e para facilitar a aplicação dos demais nutrientes, o potássio $(\mathrm{K})$ e o fósforo $(\mathrm{P})$ normalmente são também aplicados nos mesmos parcelamentos.

Viana \& Miguel (1987) aplicaram adubo contendo $\mathrm{N}$ e $\mathrm{K}$ em três parcelamentos, em quantidades iguais, numa dose contendo $20 \%$ da quantidade do adubo na primeira aplicação, $30 \%$ na segunda e $50 \%$ na terceira, não encontrando diferenças na produção com relação ao tipo de fracionamento da dose.

Parcelamentos das adubações com aplicações iniciais no período de florescimento do cafeeiro não têm promovido incrementos na produção. Isso, em parte, pode ser explicado pela insuficiência de água no solo, de modo a permitir o desencadeamento das reações necessárias para que os nutrientes passem a fazer parte da solução do solo, ficando disponíveis para as plantas. Esse resultado foi demonstrado por Matiello et al. (1983), quando aplicaram adubo nitrogenado em agosto, não obtendo o desejado. O mesmo ocorre com as adubações tardias, quando os nutrientes são adicionados ao solo, não surtindo os efeitos esperados na produção, principalmente, em virtude de os frutos já terem atingido o pleno desenvolvimento, "enchimento", e iniciando a partir daí a fase de maturação, período em que o acúmulo de nutrientes começa a decrescer (Moraes et al., 1978; Miguel, 1978).

Alguns pesquisadores têm sustentado que adubações no período chuvoso, de outubro a março, com complementação adicional tardia, mostram resultados aparentemente significativos (Mendes, 1967; Lazzarini, 1967), muito embora o efeito dessas adubações sejam de pouca relevância, apresentando produções muito semelhantes aos das adubações realizadas apenas no período chuvoso. Da mesma forma, o fracionamento das doses a serem aplicadas, divididas em partes iguais ou em proporções diferentes, não causa efeitos sobre a produção, se aplicados em dois ou três parcelamentos.

Na prática, para simplificar, a dosagem do adubo geralmente é dividida em partes iguais, e aplicada em número de parcelamentos e em épocas que podem não ser adequadas.

Assim, com este estudo objetivou-se avaliar o comportamento de cultivares comerciais de cafeeiros, de diferentes ciclos de produção, submetidas a diferentes épocas de parcelamentos das adubações, nas condições de São Sebastião do Paraíso, na região sul de Minas Gerais.

\section{MATERIAL E MÉTODOS}

O trabalho foi conduzido por dois anos na Fazenda Experimental da EPAMIG - Empresa de Pesquisa Agropecuária de Minas Gerais, em São Sebastião do Paraíso (MG), tendo sido iniciado em outubro de 1996 e conduzido até julho de 1998, quando foi realizada a última colheita.

A caracterização química e física do solo foi feita por amostragem da camada de 0 a $20 \mathrm{~cm}$ de profundidade (Tabela 1).

O solo é classificado como Latossolo Vermelho distróférrico, originalmente com vegetação de floresta tropical subperenifólia, transicional para cerrado, com textura argilo-arenosa. A área de relevo suave-ondulado com declividade média de $8 \%$ está situada à altitude de $890 \mathrm{~m}$, latitude $20^{\circ} 55^{\prime} \mathrm{S}$ e longitude $46^{\circ} 55^{\prime} \mathrm{W}$, apresenta precipitação pluvial média de $1470 \mathrm{~mm}$ distribuída de outubro a abril, temperatura média anual de $20^{\circ} \mathrm{C}$. Foram instalados quatro experimentos, cada um num talhão de uma cultivar de café: a) Mundo NovoIAC 379/19, plantada em fevereiro de 1991, no espaçamento de 4,0 m x 1,0 m; b) Icatu Precoce-IAC 3282, plantada em fevereiro de 1992, no espaçamento de 4,0 m x 1,0 m; c) Icatu Amarelo-IAC 2944, com plantio realizado em março de 1991, no espaçamento de 3,8 m x 0,7 m realizado em março de 1991, no espaçamento de 3,8 m x 0,7 m e d) Rubi-MG 1192, plantada em janeiro de 1993, no espaçamento de 3,8 $\mathrm{m}$ x 0,7 m.

Cada experimento foi constituído por 16 tratamentos (número e épocas de parcelamento da adubação), relacionados na Tabela 2 . O delineamento experimental foi o de blocos ao acaso com três repetições e parcelas de três linhas de seis plantas cada uma. Posteriormente, procedeu-se à análise conjunta dos experimentos, conforme sugere Gomes (1987), utilizando-se o Teste de Scott Knot.

A correção do $\mathrm{pH}$ do solo foi realizada em função dos teores de cálcio e magnésio resultantes da análise química, determinando a quantidade de calcário dolomitíco a ser aplicada em cada experimento.

A adubação por planta, igual para todos os tratamentos, foi de $600 \mathrm{~g}$ de adubo formulado 20-05-20, de NPK, no primeiro ano do experimento e $800 \mathrm{~g}$ do mesmo adubo no segundo ano, aplicado conforme cronograma dos tratamentos.

A aplicação em cobertura, do adubo no solo, foi realizada distribuindo-o nos dois lados das plantas, ao longo das fileiras, e do tronco até a extremidade dos ramos plagiotrópicos. 
TABELA 1 - Características físicas e químicas da amostra superficial (0 a $20 \mathrm{~cm})$ das áreas experimentais cultivadas com quatro cultivares de cafeeiros, coletadas em outubro de 1995 em São Sebastião do Paraíso (MG).

\begin{tabular}{lcccc}
\hline \multirow{2}{*}{ Características } & \multicolumn{4}{c}{ Valores } \\
\cline { 2 - 5 } & $\begin{array}{c}\text { Mundo Novo IAC } \\
\mathbf{3 7 9 - 1 9}\end{array}$ & Icatu IAC 3282 & $\begin{array}{c}\text { Icatu Amarelo } \\
\text { IAC 2944 }\end{array}$ & Rubi MG 1192 \\
\hline $\mathrm{pH}\left(\mathrm{H}_{2} \mathrm{O} 1: 2,5\right)$ & 6.4 & 4.4 & 5.6 & 6.8 \\
$\mathrm{P}\left(\mathrm{mg} / \mathrm{dm}^{3}\right)$ & 18 & 7 & 38 & 17 \\
$\mathrm{~K}\left(\mathrm{mg} / \mathrm{dm}^{3}\right)$ & 128 & 98 & 95 & 156 \\
$\mathrm{Ca}\left(\mathrm{cmol}_{\mathrm{c}} / \mathrm{dm}^{3}\right)$ & 3.5 & 1.3 & 2.3 & 4.5 \\
$\mathrm{Mg}\left(\mathrm{cmol}_{\mathrm{c}} / \mathrm{dm}^{3}\right)$ & 1.6 & 0.8 & 0.5 & 1.4 \\
$\mathrm{Al}\left(\mathrm{cmol}_{\mathrm{c}} / \mathrm{dm}^{3}\right)$ & 0.0 & 0.7 & 0.2 & 0 \\
$\mathrm{H}+\mathrm{Al}\left(\mathrm{cmol}_{\mathrm{c}} / \mathrm{dm}^{3}\right)$ & 2.3 & 3.6 & 3.6 & 1.7 \\
$\mathrm{~S}\left(\mathrm{cmol}_{\mathrm{c}} / \mathrm{dm}^{3}\right)$ & 5.4 & 2.4 & 3 & 6.3 \\
$\mathrm{t}\left(\mathrm{cmol}_{\mathrm{c}} / \mathrm{dm}^{3}\right)$ & 5.4 & 3.1 & 3.2 & 6.3 \\
$\mathrm{~T}\left(\mathrm{cmol}_{\mathrm{c}} / \mathrm{dm}^{3}\right)$ & 7.7 & 8.7 & 6.6 & 0.0 \\
$\mathrm{~m}(\%)$ & 0.0 & 23 & 6 & 79 \\
$\mathrm{~V}(\%)$ & 70 & 27 & 46 & \\
\hline Classe textural & Argilo-arenosa & & & \\
Areia & $46 \%$ & & & \\
Silt & $10 \%$ & & & \\
Argila & $44 \%$ & & & \\
\hline
\end{tabular}

TABELA 2 - Épocas de adubação utilizadas nos experimentos.

\begin{tabular}{|c|c|c|c|c|c|c|}
\hline \multirow{3}{*}{$\begin{array}{c}\text { Tratamentos } \\
1\end{array}$} & \multicolumn{6}{|c|}{ Épocas de adubação } \\
\hline & \multicolumn{6}{|c|}{ Meses } \\
\hline & - & - & Dez & Jan & $\mathrm{Fev}$ & Mar \\
\hline 2 & - & Nov & Dez & Jan & $\mathrm{Fev}$ & - \\
\hline 3 & Out & Nov & Dez & Jan & - & - \\
\hline 4 & Out & Nov & - & - & $\mathrm{Fev}$ & Mar \\
\hline 5 & Out & Nov & - & Jan & $\mathrm{Fev}$ & - \\
\hline 6 & Out & Nov & Dez & - & $\mathrm{Fev}$ & - \\
\hline 7 & - & - & - & Jan & $\mathrm{Fev}$ & Mar \\
\hline 8 & - & - & Dez & Jan & $\mathrm{Fev}$ & - \\
\hline 9 & - & Nov & Dez & Jan & - & - \\
\hline 10 & Out & Nov & Dez & - & - & - \\
\hline 11 & - & Nov & - & Jan & - & Mar \\
\hline 12 & Out & - & Dez & - & $\mathrm{Fev}$ & - \\
\hline 13 & Out & - & - & - & $\mathrm{Fev}$ & Mar \\
\hline 14 & Out & - & - & Jan & - & Mar \\
\hline 15 & Out & - & Dez & Jan & - & - \\
\hline 16 & Out & Nov & - & Jan & - & - \\
\hline
\end{tabular}

Ciênc. agrotec., Lavras. V.27, n.4, p.816-821, jul./ago., 2003 
Os cafeeiros foram sempre mantidos livres da concorrência de plantas daninhas, por capina manual. No mês de abril de cada ano, foi feita arruação, e em julho, após a colheita, a esparramação do cisco.

As colheitas foram realizadas quando os frutos apresentavam em torno de $80 \%$ no estádio de cereja, sendo a cultivar Icatu Precoce-IAC 3282 colhida em abril e as demais, em junho/julho.

Anualmente os tratamentos fitossanitários visaram basicamente ao controle de pragas e doenças que normalmente ocorrem nos cafeeiros. Nos meses de junho e julho foram realizadas aplicações de inseticidas para o controle do bicho-mineiro, e nos meses de dezembro e janeiro, para o controle da broca. $\mathrm{O}$ controle da ferrugem do cafeeiro foi realizado de forma preventiva em quatro aplicações, com fungicidas à base de cobre (oxicloreto de cobre $50 \%$ metálico).

Os micronutrientes zinco e boro foram aplicados em quatro parcelamentos (setembro, novembro, janeiro e março), na concentração de sulfato de zinco $0,5 \%$ e ácido bórico 0,3\%, em cada pulverização.

Para o estudo da produtividade, foram tomados dados de produção em todos os dezesseis tratamentos. Ao colher, determinou-se o peso dos frutos, na forma de "café da roça", por parcela. Do total, foi retirada uma amostra de $2 \mathrm{~kg}$, a qual foi seca até $11 \%$ de umidade, beneficiada, pesada, e os dados obtidos apresentados em sacas de $60 \mathrm{~kg}$ de café beneficiado por hectare.

\section{RESULTADO E DISCUSSÃO}

Foram significativas as diferenças $(\mathrm{P}>0,5 \%) \mathrm{pa}-$ ra cultivares, épocas de adubação e para a interação entre elas. Esses efeitos demonstraram que houve diferenças varietais com relação às épocas de aplicação dos fertilizantes e a cultivar Icatu Precoce-IAC 3282 foi altamente responsiva a esses efeitos (Tabela 3 ).

TABELA 3 - Valores médios da produção em sacas beneficiadas por hectare, em função das cultivares, independente das épocas dos parcelamentos da adubação, nas safras 97/98 e 98/99. UFLA, Lavras-MG, 2001.

\begin{tabular}{lcl}
\hline \multicolumn{1}{c}{ Cultivar } & Produção (sc/ha) \\
\hline Icatu Precoce-IAC 3282 & $28,78 \mathrm{a}$ & \\
Icatu Amarelo-IAC 2944 & $19,61 \quad$ b \\
Mundo Novo-IAC 379-19 & $14,83 \quad$ c \\
Rubi MG-1192 & $13,98 \quad$ d \\
\hline
\end{tabular}

Médias seguidas por letras distintas diferem entre si pelo teste de Scott \& Knott, ao nível de 5\% de probabilidade.

Não houve similaridade $(\mathrm{P}>0,5 \%)$ quanto à produção para as cultivares ensaiadas, e essa resposta pode estar condicionada a diferentes níveis de fertilidade do solo, idade das plantas ou aos espaçamentos diferenciados.

Os valores médios de produção, em sacas beneficiadas por hectare, independente da cultivar (Tabela 4 ), indicaram que as épocas correspondentes ao fracionamento das doses de adubo em quatro vezes foram as que efetivamente promoveram o melhor suprimento de nutrientes para as plantas, o que é coincidente com resultados obtidos em vários trabalhos desenvolvidos com esse objetivo (Mendes, 1967; Lazzarini, 1967; Moraes et al., 1978; Silva et al., 1984; Viana \& Miguel, 1987). Com o desdobramento dos efeitos das épocas de adubação, algumas variações surgiram, as quais foram traduzidas em opções que podem ser viabilizadas em decorrência de fatores condicionantes, principalmente o início e término do período chuvoso e disponibilidade de umidade no solo.

Os valores médios, resultantes da análise conjunta dos experimentos, constantes na Tabela 4, salientaram que três parcelamentos, com intervalos de aplicação do adubo de 60, 90 e 120 dias, como também os parcelamentos iniciados em outubro e paralisados em dezembro ou os que começaram em dezembro ou janeiro, foram os que tiveram grande influência negativa sobre a produção dos cafeeiros. Essas combinações devem ser evitadas e da mesma forma, aquelas que iniciam em outubro e são paralisadas no mês de janeiro. Isso demonstrou, de forma clara, que a adubação quando realizada em épocas inadequadas não proporcionam produções satisfatórias.

Muito embora a interação significativa, cultivares x épocas de parcelamento das adubações sugira resposta diferencial das cultivares, os resultados apresentados na Tabela 4 mostram que os quatro tratamentos, que se destacaram na média geral de produtividade, exibiram a mesma tendência nos quatro experimentos (estiveram sempre no primeiro ou segundo grupamento superior de média). Dessa forma, como sugestão geral de manejo da adubação do cafeeiro, independentemente das cultivares e do ambiente, é aceitável recomendar que esses parcelamentos da adubação tenham inicio entre outubro e dezembro e, a partir daí, não sejam interrompidos, sendo espaçados de no mínimo trinta dias, à exceção do último parcelamento, que pode ter intervalo de sessenta dias, caso a primeira aplicação tenha sido realizada em outubro. 
TABELA 4 - Valores médios da produção das cultivares Mundo Novo-IAC 379-19; Icatu Precoce-IAC 3282; Icatu Amarelo-IAC 2944 e Rubi-MG 1192, e média geral em sacas beneficiadas por hectare, em função das épocas de adubação, em 1987 96/97 e 1988. UFLA, Lavras-MG, 2001.

\begin{tabular}{|c|c|c|c|c|c|c|}
\hline Épocas de adubação & Mundo Novo & Icatu Precoce & Icatu Amarelo & Rubi & \multirow{2}{*}{\multicolumn{2}{|c|}{$\begin{array}{c}\text { Média gera } \\
22,09 \mathrm{a}\end{array}$}} \\
\hline Dez/Jan/Fev/Mar & $18,02 \mathrm{a}$ & $33,10 \mathrm{a}$ & $23,73 \mathrm{a}$ & 13,53 & & \\
\hline Out/Nov/Dez/Jan & $18,94 \mathrm{a}$ & $27,87 \quad b$ & $24,13 \mathrm{a}$ & 14,30 & $21,31 \mathrm{a}$ & \\
\hline Nov/Dez/Jan/Fev & $16,03 \mathrm{a}$ & 35,86 a & $19,35 \mathrm{~b}$ & 13,70 & $21,23 a$ & \\
\hline Out/Nov/Dez/Fev & $14,11 \mathrm{~b}$ & $31,64 \mathrm{a}$ & 23,96 a & 14,47 & $21,04 \mathrm{a}$ & \\
\hline Nov/Dez/Jan & $15,92 \mathrm{a}$ & $30,86 \mathrm{a}$ & $20,80 \mathrm{~b}$ & 14,04 & 20,40 & $\mathrm{~b}$ \\
\hline Out/Nov/Jan/Fev & $14,33 \mathrm{~b}$ & $31,20 \mathrm{a}$ & $20,03 \mathrm{~b}$ & 13,68 & 19,81 & $\mathrm{~b}$ \\
\hline Out/Nov/Jan & $15,06 \mathrm{~b}$ & $27,94 \mathrm{~b}$ & $21,93 \mathrm{~b}$ & 14,18 & 19,78 & $\mathrm{~b}$ \\
\hline Out/Dez/Jan & $16,44 \mathrm{a}$ & $28,40 \mathrm{~b}$ & $20,23 \quad b$ & 14,05 & 19,77 & $\mathrm{~b}$ \\
\hline Out/Fev/Mar & $14,26 \mathrm{~b}$ & $25,26 \quad \mathrm{c}$ & $21,60 \mathrm{~b}$ & 14,36 & 18,87 & $\mathrm{c}$ \\
\hline Out/Nov/Fev/Mar & $16,72 \mathrm{a}$ & $25,86 \quad \mathrm{c}$ & $17,93 \quad \mathrm{c}$ & 14,00 & 18,63 & $\mathrm{c}$ \\
\hline Out/Jan/Mar & $13,43 \mathrm{~b}$ & $26,66 \quad c$ & $19,13 \mathrm{~b}$ & 13,77 & 18,25 & c \\
\hline Nov/Jan/Mar & $14,23 \mathrm{~b}$ & $27,16 \quad \mathrm{c}$ & $17,26 \quad \mathrm{c}$ & 13,87 & 18,13 & $\mathrm{c}$ \\
\hline $\mathrm{Jan} / \mathrm{Fev} / \mathrm{Mar}$ & $11,53 \mathrm{~b}$ & $25,20 \quad c$ & $20,13 \quad b$ & 13,81 & 17,67 & d \\
\hline Out/Dez/Fev & $13,33 \mathrm{~b}$ & $28,10 \mathrm{~b}$ & 14,73 & 14,50 & 17,66 & $\mathrm{~d}$ \\
\hline Out/Nov Dez & $12,58 \mathrm{~b}$ & $28,21 \mathrm{~b}$ & 15,46 & 13,52 & 17,44 & $\mathrm{~d}$ \\
\hline Dez/Jan/Fev & $12,43 \mathrm{~b}$ & $26,18 \quad \mathrm{c}$ & 13,46 & 14,00 & 16,52 & $\mathrm{~d}$ \\
\hline
\end{tabular}

Médias seguidas por letras distintas diferem entre si pelo teste de Scott \& Knott, a $5 \%$ de probabilidade.

Nesse período, para a cultivar Icatu Precoce-IAC 3282, os resultados evidenciaram que o suprimento de nutrientes deve ser realizado entre outubro e fevereiro, com algumas variações (Tabela 4). Preferencialmente, a primeira aplicação de nutrientes deve ocorrer no mês de outubro, a segunda, em novembro e, finalizando, em fevereiro, com uma aplicação intercalar que pode ser efetuada em dezembro ou janeiro. Essas seriam as combinações para uma produtividade mínima desejável.

Caso ocorra adversidade climática, com retardamento do início do período chuvoso, a melhor opção seria parcelar a adubação em quatro vezes consecutivas, começando no mês de novembro e estendendo até fevereiro. Se, porventura, ainda não houver umidade suficiente no solo no mês de novembro, pode ser protelada a adubação para o início mês de dezembro, aplicando o segundo e terceiro parcelamento em janeiro e fevereiro, respectivamente, e um quarto adicional em março.

Esses resultados podem estar relacionados com a precocidade do ciclo produtivo da cultivar Icatu Precoce-IAC 3282, com o florescimento ocorrendo no mesmo período das outras cultivares, porém, com enchimento dos grãos antecipados para dezembro, enquanto as de ciclo normal e tardio têm essa fase iniciada em janeiro/fevereiro, evidenciando haver exigência nutricional diferenciada requerendo adubações, principalmente no período de maior demanda de nutrientes, que é dezembro, janeiro e fevereiro.

Comportamento semelhante foi demonstrado pela cultivar Icatu Amarelo-IAC 2944, de ciclo produtivo mais tardio que a cultivar Rubi MG-1192, necessitando de fertilização no período que vai do mês de outubro a fevereiro, em condições normais do período chuvoso. Havendo pouca disponibilidade de água no solo, o inicio da adubação pode ser prorrogado até o mês de dezembro, dividindo em quatro vezes com a última aplicada em março. Ficou evidente que essa cultivar responde favoravelmente a quatro parcelamentos.

Supostamente, devido à rusticidade apresentada pela cultivar Mundo Novo-IAC 379/19, a produção de grãos de café não foi influenciada pelo distanciamento entre épocas de adubação, desde que o suprimento de 
nutrientes seja adequado às suas necessidades. A cultivar, em questão, suportou de forma positiva os intervalos de 60 e 90 dias entre as aplicações, com produções muito próximas. Preferencialmente, a adubação para essa cultivar deve-se iniciar em outubro, prolongandose até fevereiro/março. Entretanto, fica condicionado à disponibilidade de umidade no solo o início dessa operação, que pode ser iniciada também em novembro, com três ou quatro parcelamentos consecutivos, ou em dezembro, com uma última aplicação em março.

A cultivar Rubi-MG 1192 mostrou comportamento indiferente às épocas de adubação, respondendo bem a todas as combinações. Provavelmente foi influenciada pela boa fertilidade do solo (Tabela 1) no local do experimento, que forneceu quantidades de nutrientes adequadas, e pela idade das plantas, ainda muito jovens, que sempre apresentam produções iniciais menores.

A absorção de nutrientes varia pouco entre as cultivares e é de ordem quantitativa, ou seja, os diferentes genótipos não alteram a tendência das curvas de absorção. Quando cultivares distintas apresentam simultaneamente um mesmo estádio fisiológico, a tendência de absorção é semelhante entre elas. Pesquisadores observaram que em genótipos distintos a absorção depende do desenvolvimento característico de cada cultivar, e que, em geral, a absorção dos nutrientes ocorre com maior intensidade durante as diferentes fases de desenvolvimento vegetativo e produtivo do cafeeiro. Ao se conjugar as fases fisiológicas do cafeeiro com suas exigências nutricionais nesses períodos, pode-se estabelecer um esquema objetivo e prático para as épocas mais adequadas de adubação (Carvajal, 1984).

\section{CONCLUSÕES}

O comportamento das cultivares estudadas, em relação às épocas de parcelamento da adubação as quais foram submetidas distintamente, revelou que há resposta varietal, dependendo do ciclo produtivo. A cultivar de ciclo precoce, Icatu Precoce-IAC 3282, foi a mais influenciada, evidenciando que possui exigência nutricional diferenciada. As cultivares de ciclo normal Mundo Novo-IAC 379/19 e Icatu Amarelo-IAC 2944 responderam de forma similar aos estímulos causados pelas épocas de parcelamento da adubação. A Cultivar Rubi-MG 1192, de ciclo semitardio, foi indiferente às épocas de parcelamento da adubação.

\section{REFERÊNCIAS BIBLIOGRÁFICAS}

CARVAJAL, J. F. Cafeto: cultivo y fertilización. Berna: IIK, 1984. 254 p.

GOMES, F. P. Curso de estatística experimental. 12. ed. São Paulo: Nobel, 1987. 467 p.

LAZZARINI, V.; MORAES, F. R. P. Ensaio qualitativo, quantitativo e de fracionamento de nitrogênio. In: Experimentação cafeeira: 1929 a 1963. Campinas: IAC, 1967. p. 191-194.

MATIELLO, J. B.; FIGUEIREDO, J. P.; SANTINATO, R.; BARROS, U. V. Parcelamento da adubação NPK pós colheita. In: CONGRESSO BRASILEIRO DE PESQUISAS CAFEEIRAS, 10., 1983, Poços de Caldas. Resumos... Rio de Janeiro: MIC/IBC/GERCA, 1983. p. 23-24.

MENDES, J. E. T. Ensaio de adubação nitrogenada. In: Experimentação cafeeira: 1929 a 1963. Campinas: IAC, 1967. p. 140-142.

MIGUEL, A. E. Consumo mensal de nitrogênio pelo cafeeiro: quantidade, época e modo de adubação nitrogenada. Viçosa: UFV, 1978. 13 p.

MORAES, F. R. P.; REIS, A. J.; CERVELLINI, G. S. Doses e fracionamento do cafeeiro - I: latossolo roxo de Ribeirão Preto. In: CONGRESSO BRASILEIRO DE PESQUISAS CAFEEIRAS, 6., 1978, Ribeirão Preto. Resumos... Rio de Janeiro: MIC/IBC/GERCA, 1978. p. 76-77.

SILVA, O. A.; SANTINATO, R.; FIGUEIREDO, J. P.; BARROS, U. V. Parcelamento fracionado de nitrogênio na adubação do cafeeiro. In: CONGRESSO BRASILEIRO DE PESQUISAS CAFEEIRAS, 11., 1984, Londrina. Resumos... Rio de Janeiro: MIC/IBC, 1984. p. 287-289.

VIANA, A. S.; MIGUEL, A. E. Doses e parcelamento de adubação nitrogenada e potássica para formação e produção do cafeeiro, em solo de cerrado - II. In: CONGRESSO BRASILEIRO DE PESQUISAS CAFEEIRAS, 14., 1987, Campinas. Resumos... Rio de Janeiro: MIC/IBC, 1977. p. 249-252. 